\title{
Computerized Assessment of the Functionality of the Human Upper Limb: New Approaches
}

\author{
Viorel Gheorghe, Daniel Miu, Doina Bucur, Diamanta Mihaela Visan, and Nicolae Daniel Simion
}

\begin{abstract}
Musculoskeletal disorders are the most frequent health problem of active population in industrialized countries, with considerable costs and impact on quality of life. Getting a precise and fast diagnosis generates a properly applied treatment with favorable effects. Mobility of the upper limb can be determined in medical practice through a large number of devices. Taking into account their disadvantages, two simple systems were proposed from a constructive point of view with a low but good efficiency. In order to determine which of the two projected systems is most effective, a comparison was made between the chosen systems and the upper limb motion analysis system, namely Vicon Mx. One of the systems has been highlighted in relation to the other, with a lower measurement error due to the IMU sensors that the system has in its composition. The equipment is easy to use for the medical environment, it is comfortable and fast for the patient, so this proposed system is the one that would be needed in this field, its realization contributing to the technological advance of the current systems.
\end{abstract}

Index Terms-Electrogoniometer, upper limb range of motion, orthosis.

\section{INTRODUCTION}

Musculoskeletal pathologies are the most frequent health problem of the active population in industrialized countries with considerable costs and impact on quality of life, leading to functional impotence and patient inability to perform normal activities [1], [2]. Under these circumstances, clinical examination is a mandatory and important step towards a precise and rapid diagnosis that will generate a properly applied treatment with favorable effects [3]. Devices that measure the degree of mobility of the upper limb have the role of shortening diagnosis time, and at the same time helping the specialist to make a precise diagnosis.

Mobility of the upper limb can be determined in medical practice by means of hand-held devices called goniometers or three-dimensional systems called inertial measurement units systems (IMU) that show in real time the orientation of a device using sensors data sets and several processing operations. Smartphones, video motion or optoelectronic motion analysis (Quintic, VICON, etc.) can also be used.

Obtaining fast measurements, made easily and that can be subsequently confirmed by paraclinical tests in order to obtain a diagnosis of certainty, is indispensable to the medical act, so it has been decided to develop a device that will remove the disadvantages of the existing equipment.

Manuscript received September 28, 2017; revised October 28, 2017

Viorel Gheorghe, Daniel Miu, Doina Bucur, Nicolae Daniel Simion are with Polytechnic University of Bucharest, Romania (e-mail: diavisan@yahoo.com).

Diamanta Mihaela Visan is with National College "Mihai Eminescu", Romania.

\section{METHODOLOGY}

Two systems based on different methods of measuring the mobility of the upper limb have been designed. The first variant of the system (Fig. 1), which assures the mobility of the upper limb, is a simple method by which the patient has positioned the upper limb between the elbow holder (4) and the sensor support (2).

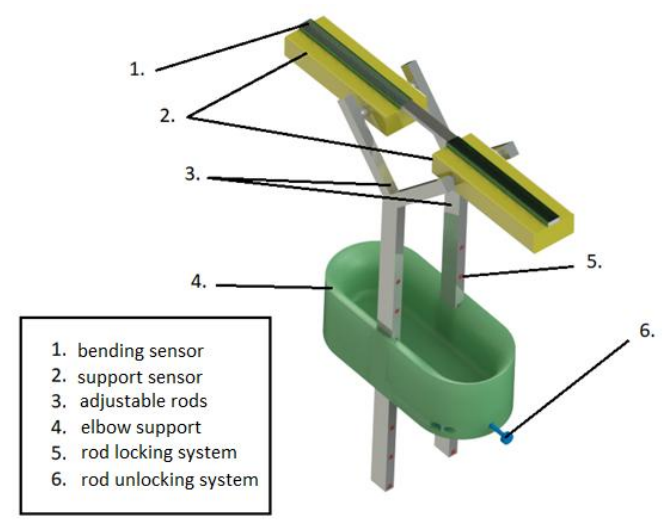

Fig. 1. System design variant 1.

The system has adjustable rods (3) so that after the patient has attached the device to the arm, it adjusts according to its size and remains locked until unlocked by pulling the rod (6) when the measurement process is complete.

The bending sensor is the one that measures the angle between the arm and the patient's forearm, information which is transmitted to a computer where, in real time, the movement of the arm and the measured angle will be displayed, the next step being to enter the patient's data (name, age, sex, diagnosis) to be saved and attached to their medical record.

The second variant of the system is shown in Fig. 2.

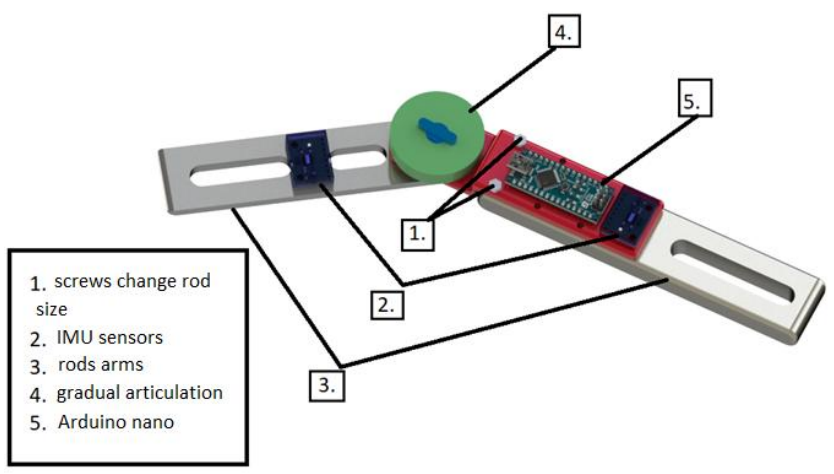

Fig. 2. System design variant 2.

The designed system has its primary purpose to determine with high precision the mobility of the upper limb. The 
system is composed of an orthosis (forearm metal rod, upper arm metal rod), IMU sensors (2), graduated joint (4), orthosis length adjusting screws (Fig. 2). The orthosis will be positioned on the upper limb as follows: the two rods (3) will be attached to the forearm and upper arm and the graduated joint (4) will be located at the elbow level. On the upper arm rod, an Arduino development board (5) is mounted along with a small battery pack.

The clamping of this orthosis on the upper limb is accomplished by placing on the anterior face of the orthosis sleeves provided with grippers (Fig. 3) which will be inserted through the spaces within the two rods. In this way, the holding becomes very comfortable for the patient, but also ensures a precise positioning of the orthosis for a more accurate measurement and, implicitly, a lower measurement error.
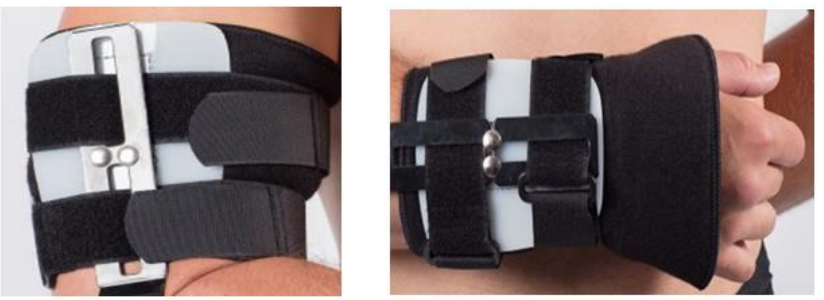

Fig. 3. Clamping sleeve arm and forearm attachment sleeve.

This joint also has a graduated scale similar to the goniometer, with the aim of providing an approximate measurement that will be compared with the digital measurement itself in order to avoid the various measurement errors of the digital system without the qualified personnel observing this defect. The graduated system will then be used to calculate the real deviation of a system of this type from the digital one to be able to highlight the benefits of the designed system.

On the orthosis are mounted the two IMU (Fig. 3) made by Adafruit, an Arduino development board and a battery pack.

These sensors will communicate with a Nano Arduino board, chosen due to the small size required for such a system. A part of the necessary data processing will be carried out at this level, and the processed information will be transferred to the computer via a Bluetooth connection, having also a tethered backup connection.

By using human-body IMUs, the data received from these sensors can be translated into real-time 3D representations.

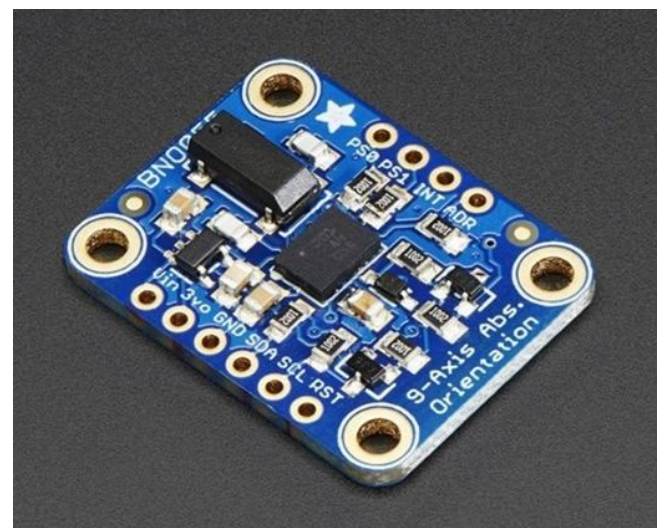

Fig. 3. BNO055 IMU sensor.

The IMU is based on the System in Package - BNO055, developed by Bosch Sensortec and integrates a triaxial 14-bit accelerometer, a triaxial 16-bit gyroscope with a range of \pm 2000 degrees per second, and a triaxial geomagnetic sensor. The nine sensor gathered data is internally processed by the BNO055 with the help of a 32-bit microcontroller and a proprietary BSX3.0 FusionLib software which is capable to deliver absolute orientation of the IMU at a rate of up to $100 \mathrm{~Hz}$. The communication between the two IMUs and the Arduino development board is achieved through an $\mathrm{I}^{2} \mathrm{C}$ communication protocol.

The controller board is an Arduino Nano development board, with a small foot print (roughly $43 \times 15 \mathrm{~mm}$ ) governed by a microcontroller Atmel ATMega 328P, operating at a nominal frequency of $16 \mathrm{MHz}$.

The mode of operation of the projected system can be described as follows: the IMU sensors, using all its nine fused data stream from the sensors (accelerometers, gyroscopes, magnetometers), detect the movements of the upper limb. The data is sent to the Arduino development board Nano that will partially process the information and pass it through the Bluetooth communication module, (untethered operation mode - preferable option) or through a cable to a PC (tethered operation mode). With the help of a Java based application is then graphically rendered the real-time motion of the upper limb and its mobility degrees, which makes possible for the physician to pronounce the diagnosis. (Fig. 4)

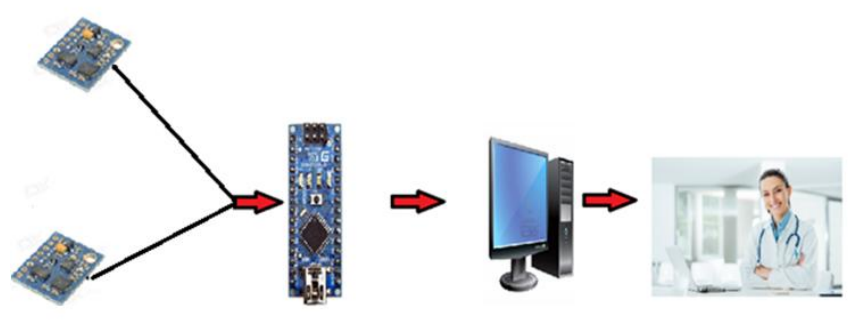

Fig. 4. Graphical presentation of the operation mode

The Java interface (fig. 5) contains the necessary needed editable and non-editable fields, along with a visual representation of the taken measurement.

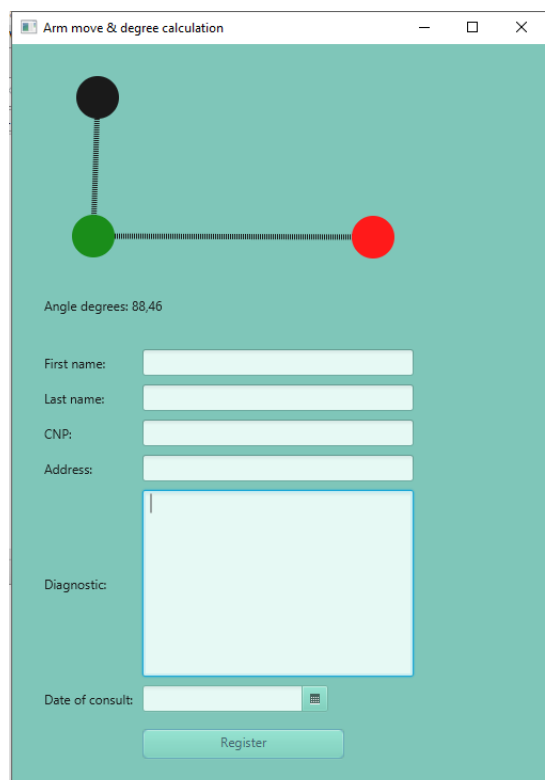

Fig. 5. Java based interface.

One of the goals of the interface is to be easy to comprehend and use without an extensive specialized 
training. In order to simplify the physicians work, each measurement of the upper limb's mobility can be saved, along with the patient's data (first name, last name, address, personal identification number CNP, address, and diagnostic) and the date when the consultation has been made.

For testing purposes, that can lead to a final product a simpler solution has been used (Fig. 6).

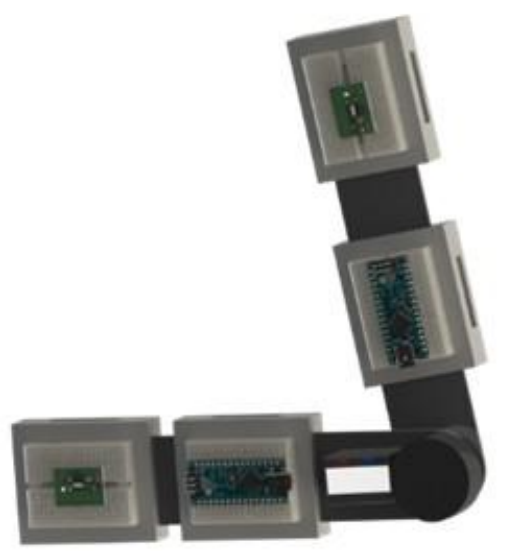

Fig. 6. Test platform, a CAD render.

The testing platform has a design as similar as possible with the second variant of the measurement system. A lot of its components are produced by 3D printing (FDM - Fused deposit technology), that ensures fast production time intervals and a satisfactory mechanical precision.

The platform is endowed with the same IMU units, graduated joint and it has slight changes regarding the way in which orthosis is attached to the upper limb.

The hardware has an extra Arduino Nano board for easy processing of the data and the operation mode in this case is a tethered one, having a USB data cable connected for communication and electrical power necessities.

Validation of the testing platform is being done by an optoelectronic equipment VICON MX. This is a multi-camera system with embedded stroboscope. The camera's strobe emits radiation in the near-infrared area, and the camera's sensor receives the signals reflected by the IR reflexive markers.

The 2D information received from the cameras is transmitted to a central computer that digitizes in real time the received data and performs the three-dimensional reconstruction of markers positions. (Fig. 7)

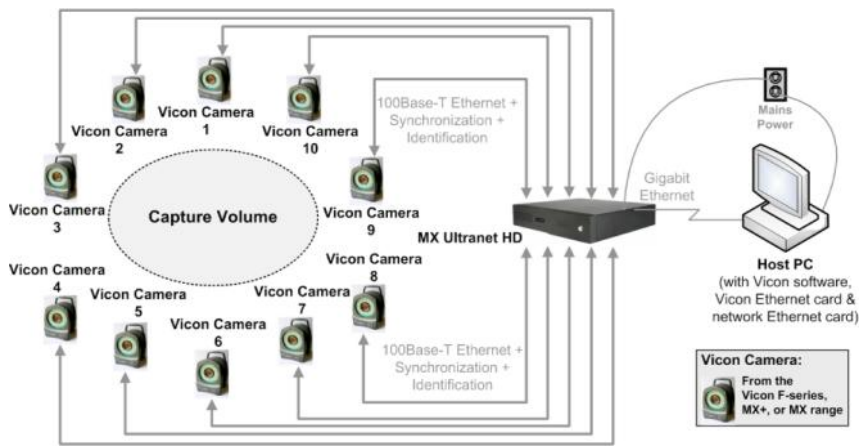

Fig. 7. Optoelectronic system Vicon MX[14].

The system is composed by $7 \mathrm{MX} 3$ + cameras that record at $659 \times 493$ pixels in grayscale, with up to 240 frames per second. The cameras are equipped with several high-speed controllers that perform real-time image processing. If the capture resolution decreases, cameras can capture up to 1000 frames per second. The communication between the systems' elements are achieved through an Ultranet MX Gigabit communication network.

Fig. 8 presents several types of infrared reflexive merkers used in Vicon MX system.
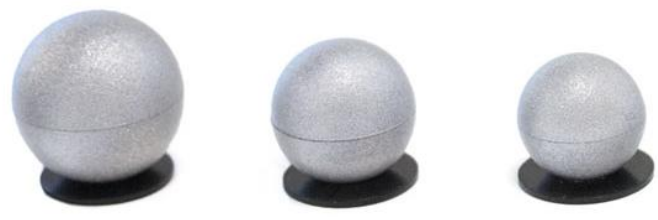

Fig. 8. Vicon reflexive markers.

\section{RESULTS AND ANALYSIS}

In order to determine which of the two projected systems is the most efficient, a comparison was made between the chosen systems and the upper limb mobility determination system, namely Vicon Mx.

As a result of these comparisons was found that the efficiency of the system projected in version 2 is higher because it is highlighted against the first system with a lower measurement error (Table I).

TABLE I: COMPARISON BETWEEN THE DESIGNED SYSTEMS

\begin{tabular}{|c|c|c|c|}
\hline Required features & Vicon Mx & $\begin{array}{c}\text { Designed } \\
\text { system 1 }\end{array}$ & $\begin{array}{c}\text { Designed } \\
\text { system 2 }\end{array}$ \\
\hline $\begin{array}{c}\text { Small measurement } \\
\text { error }\end{array}$ & $\mathrm{X}$ & $\mathrm{X}$ & $\mathrm{X}$ \\
\hline $\begin{array}{c}\text { Easy to use for } \\
\text { physician }\end{array}$ & & $\mathrm{X}$ & $\mathrm{X}$ \\
\hline Portability & & $\mathrm{X}$ & $\mathrm{X}$ \\
\hline Low cost & $\mathrm{X}$ & $\mathrm{X}$ & $\mathrm{X}$ \\
\hline $\begin{array}{c}\text { Comfortable for } \\
\text { patient }\end{array}$ & $\mathrm{X}$ & $\mathrm{X}$ & $\mathrm{X}$ \\
\hline Flexibility & $\mathrm{X}$ & $\mathrm{X}$ \\
\hline $\begin{array}{c}\text { Measuring } \\
\text { rapidness }\end{array}$ & & & \\
\hline
\end{tabular}

Thus, for further testing the attention has been focused on the second variant of the system, more exactly on the developed testing platform.

Tests have been performed for assessing the degree of mobility only for the forearm with respect to the upper arm. The Vicon system and the hardware of the test platform have taken measurements simultaneously.

The markers necessary for the Vicon system assessment are positioned as follows:

- A, positioned at the forearm level;

- B, positioned at the upper arm level;

- O, positioned at the elbow level. (Fig. 9)

These positions were specifically chosen having in mind an accurately description of the elbow movement.

For starters both system were calibrated and afterwards the angle between the forearm and upper arm was measured. Three values of the angle have been taken under consideration 0,45 and 90 degrees.

For each measurement the orthosis was moved in order to reflect the pre-established test values. The graduated joint value was taken under consideration when the preset test values were achieved. 
For a proper evaluation, the data taken from the Vicon MX system was the raw tridimensional coordinates of the three marked points A, B and O. Afterwards, at the PC level the data was transformed into degree angle values.

The test platform for each pre-established value was given two sets of orientation Euler angles, one for the forearm and the second one for the upper arm. Similar with the Vicon system the necessary flexion angles were computed, again with the help of the PC.

The first verified position is the zero, anatomic one. In this situation the upper arm and the forearm describe a line. As can be seen in Fig. 10, the arm is in the designated position and the virtual space of the Vicon system respects the reality.

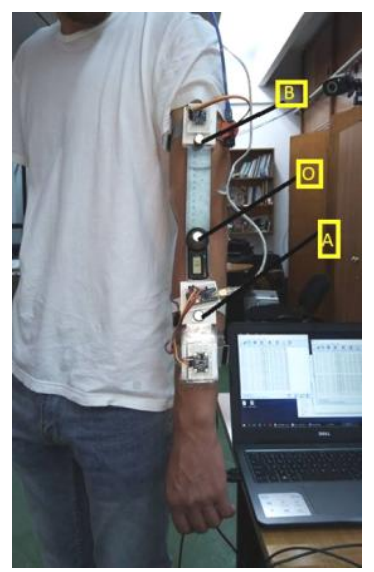

Fig. 9. Test equipment with Vicon markers.

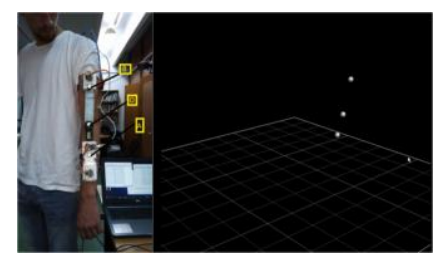

Fig. 10. First test measurement $(0 \mathrm{deg})$

Next, the upper arm is flexed at 45 degrees. As in the previous situation the virtual environment is in close resemblance with the actual posture. (Fig. 11)

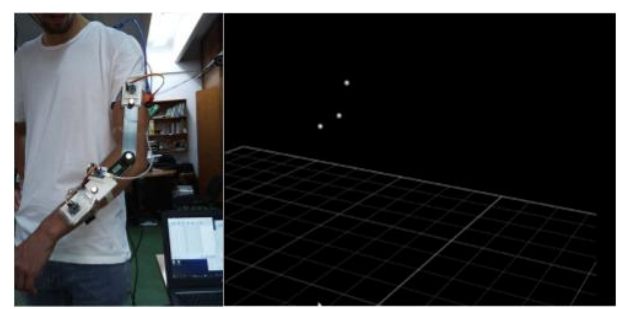

Fig. 11. Second test measurement (45 deg).

The last set of measurement were taken with a flexion of 90 degrees between the upper arm and the forearm (Fig. 12). In the virtual space, at this time, can be seen besides the points $\mathrm{A}, \mathrm{B}$ and $\mathrm{O}$ the orientation of two of the Vicon system cameras.

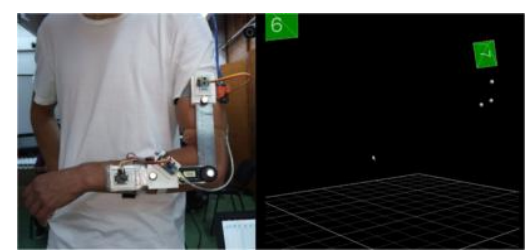

Fig. 12. Third test measurement (90 deg)
In Table II, the obtained values are shown. The Vicon MX presents in the test setup an error ranging from 1.63 to 4.05 degrees. The errors might occur due to a slight misposition of the reflective markers mainly caused by manufacturing tolerances, operator errors, and data processing.

The test platform based on two IMUs obtained an error between 2.95 to 10.2 degrees, explained by misalignment of the IMUs with respect to the designated axis, due to montage and manufacturing imperfections. Also, the processed data algorithms might be improved for a higher accuracy.

Given the fact the form and the degree of movement of the human upper limb, both systems tested give satisfactory (test platform) to excellent results (Vicon MX).

TABLE II: THE OBTAINED FLEXION ANGLES

\begin{tabular}{|c|c|c|}
\hline $\begin{array}{c}\text { Graduated joint } \\
{[\mathrm{deg}]}\end{array}$ & $\begin{array}{c}\text { Vicon MX } \\
{[\mathrm{deg}]}\end{array}$ & $\begin{array}{c}\text { IMU based system } \\
{[\mathrm{deg}]}\end{array}$ \\
\hline 0 & 3.44 & 7.75 \\
\hline 45 & 49.05 & 47.95 \\
\hline 90 & 88.37 & 79.80 \\
\hline
\end{tabular}

\section{CONCLUSIONS}

The paper aims to study the development of equipment that will contribute to the technological advance of the current systems. Taking into account their disadvantages, two simple systems were proposed from a constructive point of view with a low cost but good efficiency.

The study presented concluded that both designed variants are viable, the second designed system being considered more efficient and flexible than the first one. The devices tend to be easy to use for the medical environment, without requiring specialized training and most important, it is comfortable for the patient.

The tests done on a simplified version of the second variant of the proposed system using a reference optoelectronic system, Vicon MX were satisfactory. This fact corroborated with the system flexibility and upgrading possibilities can make a patient friendly system for measuring an extensive range of upper limb functionalities.

\section{REFERENCES}

[1] J. L. Kelsey, A. White, H. Pastides, GE Jr Bisbee, "The impact of musculoskeletal disorders on the population of the United States," $J$ Bone Joint Surg Am. 1979 Oct, vol. 61, no. 7, pp. 959-964.

[2] P. K. Hembecker, C. Reis, A. C. Konrath, A. Gontijo L, D Merino, "Investigation of musculoskeletal symptoms in a manufacturing company in Brazil: A cross-sectional study," Braz J Phys Ther, 2017.

[3] T. Petersen, M. Laslett, and C. Juhl, "Clinical classification in low back pain: best-evidence diagnostic rules based on systematic reviews," BMC Musculoskelet Disord, 2017, vol. 18, no. 1.

[4] J. O. Woodman, "An introduction to inertial navigation," Technical Report, pp. 5-21, University of Cambridge August 2007.

[5] D. P. Groves, "Principles of GNSS, inertial and multisensor integrated navigation systems," pp. 3-159, Artech House, 2008.

[6] L. R. Gajdosik and R. W. Bohannon, "Clinical measurement of range of motion," Review of Goniometry Emphasizing Reliability and Validity," 1987, vol. 67, 1867-1872.

[7] B. Behnoush, N. Tavakoli, E. Bazmi, F. Nateghi, P. Shahi, M. H. A. Okazi, and T. Mokhtari, "Smartphone and universal goniometer for measurement of elbow joint motions: A comparative study," Asian J Sports Med, 2016, vol. 11, vol. 7, no. 2.

[8] M. J. Mullaney, M. P. McHugh, C. P. Johnson, and T. F. Tyler, "Reliability of shoulder range of motion comparing a goniometer to a digital level," Physiother Theory Pract, 2010, vol. 26, no. 5, pp. 327-333.

[9] M. R. Pourahmadi, T. I. Ebrahimi, Sarrafzadeh J, Bahramian M, Mohseni-Bandpei, F. Rajabzadeh, M. Taghipour, "Reliability and concurrent validity of a new iPhone ${ }^{\circledR}$ goniometric application for 
measuring active wrist range of motion: A cross-sectional study in asymptomatic subjects," J Anat. 2017 Mar, vol. 230, no. 3, pp. 484-495.

[10] G. Sacco, J M. Turpin, A. Marteu, C. Sakarovitch, B. Teboul, L. Boscher, P. Brocker, P. Robert and O. Guerin, "Inertial sensors as measurement tools of elbow range of motion in gerontology," Clin Interv Aging, 2015, vol. 10, pp. 491-497;

[11] A. Wong, Radiocarpal Joint.

[12] B. P Thomas and R. Sreekanth, "Distal radioulnar joint injuries," Indian J Orthop, 2012, vol. 46, no. 5, pp. 493-504.

[13] M. L. Cuadrado, J. A. Arias, M. A. Palomar, and R. Linares, "The pyramidal tract: New pathways," Rev Neurol, 2001.

[14] Vicon. [Online]. Available: http://www.vicon.com.

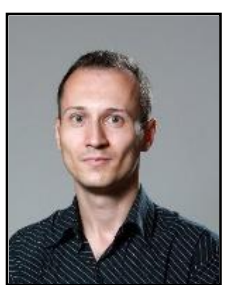

Viorel Gheorghe has a $\mathrm{PhD}$ degree in Engineering Mechanics (University Politehnica of Bucharest (UPB), 2012), and a BSc and master degree in mechatronics (UPB, 2003, 2004).

$\mathrm{He}$ is a senior lecturer at the Department of Mechatronics and Precision Mechanics, Faculty of Mechanical Engineering and Mechatronics (UPB) where he teaches mechatronics, robotics, pneumatics and hydraulics and supervises BSc and MSc students.

His interest research interests lie in the area of optomechatronics and biomimetic robots. Current research deals with electromagnetic actuated MEMS, and bioinspired mobile robotic structures.

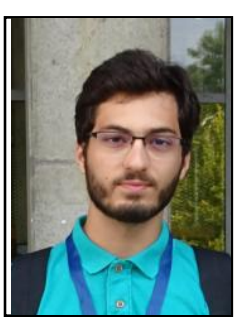

Daniel Miu is student at the Faculty of Engineering in Foreign Languages, Polytechnic University of Bucharest. $\mathrm{He}$ is passionate about biomechanics research, being preoccupied about discovering new ways to improve sports performance. $\mathrm{He}$ is the initiator of the study on Krav-Maga martial art, study that could be extended to other self-defense sports. Previous experience recommends him as a good competitor, competing in multiple olympiads on computer science.

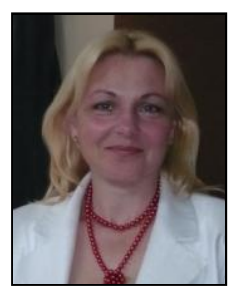

Diamanta Mihaela Visan is a graduate of the Faculty of Physics, Bucharest University. The diploma project was focused on mathematical and physical modeling in biology. She is also graduate of the Faculty of Economics and a Master of Human Resource Management. She is passionate about biomechanics research, which is an area that can answer many problems faced by mankind. Previous experience has focused on didactic activities and teams management.

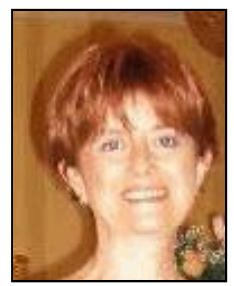

Doina Bucur has a $\mathrm{PhD}$ degree in Enginering Mechanics (Politehnica University of Buchares (UPB), 2002), and a BSc degree in precision mechanics (UPB, 1982).

She is senior lecturer at the Department of Mechatronics and Precision Mechanics and of Faculty of Medical Engineering (UPB) where she teaches Biomechanics, Prosthetics and Orthotics and Design of medical equiment, supervises $\mathrm{BSc}$ and $\mathrm{MS}$ students. After graduation she worked in a company for manufactruring of electrical equipment (1982-1984) and started her activity in UPB in 1984 as a Research Scholar, asistant lecturer (1985-1992) and senior lecturer in present. She was awarded two scholarships (Tempus-1997, Soros-2001) at Oxford Orthopaedic Engineering Centre, UK, and in 2001 to Politehnica of Milan, where she attended a Course on Motion Analysis. She is member of Romanian Society for Mechatronics Engineering (SROMECA), Romanian Asociation of Standardization (ASRO) where she is also secretary of National Technical Committees for Standardization 377

Ms. Doina Bucur's main research interests include biomechanics and upper limb prostheses. 